\title{
Bone Metastases in Newly Diagnosed Colorectal Cancer by Tumor Location: a Population-based Study
}

Lin Yang ( $\square$ yanglin1nfyy@smu.edu.cn )

Southern Medical University Nanfang Hospital https://orcid.org/0000-0003-4544-8071

Shousheng Liu

Sun Yat-sen University Cancer Center

Qiankun Xie

Southern Medical University Nanfang Hospital

Wenzhuo He

Sun Yat-sen University Cancer Center

Chang Jiang

Sun Yat-sen University Cancer Center

\section{Bei Zhang}

Sun Yat-sen University Cancer Center

\section{Liangping Xia}

Sun Yat-sen University cancer center

\section{Primary research}

Keywords: colorectal cancer, bone metastasis, tumor site, prognosis

Posted Date: February 12th, 2020

DOI: https://doi.org/10.21203/rs.2.23358/v1

License: (우 (i) This work is licensed under a Creative Commons Attribution 4.0 International License. Read Full License 


\section{Abstract}

Purpose

Bone metastasis (BM) in colorectal cancer (CRC) is rare and data on population-based incidence is lacking. We aimed to evaluate the incidence of BM and the risk factors for BM in CRC.

Methods

The Surveillance, Epidemiology, and End Results (SEER) database was interrogated as part of the analysis. We used multivariable logistic and Cox regression analyses to determine predictors for the presence of BM at diagnosis, and the elements associated with poor survival. Kaplan-Meier analysis was used to estimate the survival difference among subgroups.

Results

A total of 166,388 adult patients were identified between 2010 and 2013 as being diagnosed with CRC. Of these, 1,659 patients developed BM representing $1 \%$ of the entire CRC cohort and $5.5 \%$ of patients had metastatic disease. The median survival period of patients with BM was merely 11.3 months. We found that extracranial metastases number and tumor site were associated with BM at CRC diagnosis and these parameters were also associated with poorer survival in the BM cohort. Unlike other solid tumors, patients with bone metastasis had poorer survival and the median survival time (MST) was significantly shortened for single metastasis disease or when lung and liver metastasis were present.

\section{Conclusions}

The findings of this study clearly reveal the incidence and prognosis for patients with BM at time of CRC diagnosis. Our findings lend support for positive treatment of BM without other organ metastasis.

\section{Background}

Colorectal cancer is the third most common type of cancer worldwide[1-3]. Approximately $20 \%$ of patients with colorectal cancer (CRC) have metastatic disease at the time of diagnosis[4]. The liver is the most common site of metastatic disease from CRC and between 15 to $20 \%$ of patients present with synchronous liver metastases[5, 6]. The incidence of lung metastasis in CRC is approximately $10 \%[7]$. Compared with liver or lung metastasis in patients with CRC, bone metastasis is relatively rare and approximates between 10-15\% [8].

Patients with bone metastasis are often diagnosed because of skeletal-related events (SREs), such as severe bone pain, spinal cord compression, pathologic fractures, and hypercalcemia[9]. Since SREs often represent the late events associated with bone metastasis, patients with bone metastasis often experience lower quality of life and poor physical condition. Therefore, the early diagnosis of bone metastasis is imperative. However, the characteristics associated with bone metastasis at diagnosis of CRC are not yet clear[10,11]. 
The majority of patients with metastatic CRC ( $\mathrm{mCRC}$ ) have incurable disease. Studies have reported that the prognosis of patients with bone metastasis is very poor, with a 5 -year survival rate less than $5 \%[12,13]$. However, survival in advanced CRC has improved because of concomitant advances in precision medicine, increased acceptance of metastasectomy, and the introduction of immunotherapy[14, 15]. There may be different survival benefits based on the site of disease metastasis and the different therapeutic options that are now available.

Our aim in this study was to evaluate characteristics of bone metastasis from CRC and to clarify the prognostic factors associated with survival in these patients.

\section{Methods}

The data were retrieved from the Surveillance, Epidemiology, and End Results (SEER) program of the National Cancer Institute SEER database, USA, after 2010. This included whether bone metastasis was confirmed at the diagnosis of CRC. The datasets are available https://seer.cancer.gov/ in the SEER dataset repository.

The "TNM" staging was determined by the "extent of cancer" at the initial diagnosis using the 7th AJCC edition. Pathology was stratified into adenocarcinoma (AC), or mucinous adenocarcinoma (MC), or other. Tumor grade was recorded as well differentiated, moderately differentiated, poorly differentiated, or undifferentiated. Only patients with a detailed tumor location were included in our analysis, including rightsided colonic cancer (RCC) (cecum, ascending colon, hepatic flexure, and transverse colon), left-sided colon cancer (LCC; splenic flexure, descending colon, and sigmoid colon), rectosigmoid cancer (RSC; rectosigmoid junction and rectum), and appendix cancer[16].

Race/ethnicity was categorized as previously described[17]. The SEER 9 dataset categorized ethnicity as white, African-American, Native American/Alaska Native, Asian/Pacific Islander, and unknown. The extent of disease was evaluated by the sites of metastasis and number of bone, lung, and liver metastases at diagnosis that were entered in the SEER database.

Patient survival was recorded by overall survival and CRC-cause specific death. The follow-up time was computed by the time of first diagnosis of CRC until the last follow-up, death, or end of the study, whichever occurred first. To assess CRC-specific mortality, we used Fine and Gray's competing risk regression[18].

Patients that were found to have bone metastasis 6 months after the diagnosis of CRC or bone metastasis by autopsy were excluded. Thus, there were 166,388 patients diagnosed with CRC that were included in our analysis. Of these CRC patients, 1,659 were diagnosed with bone metastasis.

\section{Statistical Analysis}

Multivariable logistic regression was used to determine which characteristic was associated with the presence of bone metastasis at diagnosis among CRC patients or the M1 cohort (Patients with distant metastasis at the initial diagnosis). Multivariable Cox regression was performed to identify covariates associated with poor survival. The Kaplan-Meier method was used to estimate the survival difference between the subgroups. 
Two-sided $\mathrm{P}$ values $<0.05$ were considered statistically significant. Statistical analysis was performed using SAS, version 9.2 (SAS Institute, Cary, NC, USA).

\section{Results}

\section{Incidence}

There were 166,388 patients diagnosed as having CRC in our analyses. This included $41.5 \%$ with RCC, $26.8 \%$ with LCC, and $31.7 \%$ with RSC, respectively. There were 30,203 patients with metastasis disease at any site and of these, there were 12,396 (41.0\%), 8,758 (29.0\%), and 9,049 (30.0\%) patients with RCC, LCC, and RSC, respectively. The distribution of patients with $\mathrm{CRC}$ and identified bone metastases at diagnosis are provided in Table 1, as stratified by tumor location. Among the entire cohort, 1,659 patients presented with bone metastases at diagnosis, representing $1 \%$ of the entire study and $5.5 \%$ of the subset with metastatic disease at any site. Bone metastasis numbers were $563(0.8 \%), 407$ (0.9\%) and 689 (1.3\%) for RCC, LCC, and RSC, respectively. RSC had the highest incident percentage of bone metastasis ( $1.3 \%$ of the entire cohort; $7.6 \%$ of the metastatic subset).

Table 1

Incidence and median survival of patients diagnosed with CRC and bone metastases

\begin{tabular}{|c|c|c|c|c|c|c|c|}
\hline \multirow[t]{2}{*}{ Subtype } & \multicolumn{3}{|c|}{ Patient numbers } & \multicolumn{2}{|c|}{$\begin{array}{l}\text { Percentage Incidence } \\
\text { of bone Metastases } \\
\text { (\%) }\end{array}$} & \multirow{2}{*}{$\begin{array}{l}\text { Survival } \\
\text { Among } \\
\text { Patients } \\
\text { With Bone } \\
\text { Metastases, } \\
\text { Median } \\
\text { (IQR), Mo }\end{array}$} & \multirow{2}{*}{$\begin{array}{l}\text { Survival } \\
\text { Among } \\
\text { Patients } \\
\text { Without Bone } \\
\text { Metastases, } \\
\text { Median (IQR), } \\
\text { Mo }\end{array}$} \\
\hline & $\begin{array}{l}\text { With } \\
\text { CRC }\end{array}$ & $\begin{array}{l}\text { Among } \\
\text { Subset } \\
\text { With } \\
\text { Metastatic } \\
\text { Disease }\end{array}$ & $\begin{array}{l}\text { Bone } \\
\text { Metastases }\end{array}$ & $\begin{array}{l}\text { Among } \\
\text { Entire } \\
\text { cohort }\end{array}$ & $\begin{array}{l}\text { Among } \\
\text { Subset } \\
\text { With } \\
\text { Metastatic } \\
\text { Disease }\end{array}$ & & \\
\hline $\begin{array}{l}\text { Whole } \\
\text { cohort }\end{array}$ & 166,388 & 30,203 & 1,659 & $1 \%$ & $5.5 \%$ & $\begin{array}{l}11.37 \\
(10.54- \\
12.19)\end{array}$ & $\begin{array}{l}19.91 \\
(19.65- \\
20.17)\end{array}$ \\
\hline $\mathrm{RCC}$ & 69,065 & 12,396 & 563 & $0.8 \%$ & $4.5 \%$ & $\begin{array}{l}8.34(7.38- \\
9.40)\end{array}$ & $\begin{array}{l}\text { 16.54(16.16- } \\
19.9)\end{array}$ \\
\hline LCC & 44,548 & 8,758 & 407 & $0.9 \%$ & $4.6 \%$ & $\begin{array}{l}12.43 \\
(10.62- \\
14.24)\end{array}$ & $\begin{array}{l}22.29 \\
(21.79- \\
22.29)\end{array}$ \\
\hline RSC & 52,739 & 9,049 & 689 & $1.3 \%$ & $7.6 \%$ & $\begin{array}{l}12.98 \\
(11.61- \\
14.34)\end{array}$ & $\begin{array}{l}22.23 \\
(21.74- \\
22.72)\end{array}$ \\
\hline
\end{tabular}


When multivariable logistic regression (Table 2) was performed on patients with metastatic cancer, being male (Female vs. male, OR $=0.854,95 \% \mathrm{Cl}, 0.762-0.958, \mathrm{P}<0.001$ ), advanced stage (N1 vs. N0, OR $=1.278$, $95 \% \mathrm{Cl}, 1.102-1.483, \mathrm{P}=0.001 ; \mathrm{N} 2$ vs. $\mathrm{NO}, \mathrm{OR}=1.548,95 \% \mathrm{Cl}, 1.548-1.949, \mathrm{P}<0.001$ ), mucinous carcinoma (mucinous vs. adenocarcinoma, $\mathrm{OR}=1.447,95 \% \mathrm{Cl}, 1.142-1.832, \mathrm{P}=0.002$ ), poorly or undifferentiated (poorly vs. well, $\mathrm{OR}=1.024,95 \% \mathrm{Cl}, 1.186-2.309, \mathrm{P}=0.003$; poorly differentiated vs. well-differentiated, $\mathrm{OR}=1.913$, $95 \% \mathrm{Cl}, 1.244-2.941, \mathrm{P}=0.003$ ), and non-receipt of surgery (Yes, No, OR $=0.363,95 \% \mathrm{Cl}, 0.309-0.426, \mathrm{P}<$ 0.001 ) were all associated with significantly greater odds of having bone metastases at diagnosis. Notably, patients with brain metastasis were at highest risk of bone metastasis compared with other organ metastasis (brain metastasis (Yes vs. No, OR $=6.035,95 \% \mathrm{Cl}, 2 . .848-12.787, \mathrm{P}<0.001$ ), lung metastasis (Yes vs. No, OR = $3.881,95 \% \mathrm{Cl}, 1.873-8.040, \mathrm{P}<0.001$ ), and liver metastasis (Yes vs. No, $\mathrm{OR}=2.168,95 \% \mathrm{Cl}, 1.055-4.452, \mathrm{P}=$ $0.035)$ ). RAS cancer patients had higher risk of bone metastasis compared with $\mathrm{RCC}(\mathrm{HR}=1.600,95 \% \mathrm{Cl}$, 1.427-1.795, $\mathrm{P}<0.001)$. Patients who had more organ metastatic lesions were at increased risk of bone metastasis (more extra-bone metastatic lesions ( 1 vs. $0, \mathrm{OR}=3.826,95 \% \mathrm{Cl}, 1.662-8.808, \mathrm{P}=0.002 ; 2$ vs. 0 , $\mathrm{OR}=6.557,95 \% \mathrm{Cl}, 5.641-7.623, \mathrm{P}<0.001 ; 3$ vs. $0, \mathrm{OR}=8.191,95 \% \mathrm{Cl}, 6.381-10.514, \mathrm{P}<0.001))$. 
Table 2

Multivariable Logistic Regression for the Presence of Bone Metastases at Diagnosis of CRC.

\begin{tabular}{|c|c|c|c|c|c|c|}
\hline \multirow[t]{2}{*}{ Patient characteristic } & \multicolumn{2}{|l|}{ Patients } & \multicolumn{2}{|c|}{ Among Entire cohort } & \multicolumn{2}{|c|}{$\begin{array}{l}\text { Among cohort with } \\
\text { metastasis }\end{array}$} \\
\hline & Patients & $\begin{array}{l}\text { Bone } \\
\text { metastasis }\end{array}$ & OR $(95 \% \mathrm{Cl})$ & $\begin{array}{l}P \\
\text { value }\end{array}$ & $\begin{array}{l}\text { OR } \\
(95 \% \mathrm{Cl})\end{array}$ & $\begin{array}{l}P \\
\text { value }\end{array}$ \\
\hline \multicolumn{7}{|l|}{ Sex } \\
\hline Male & 87,092 & 830 & & & & \\
\hline Female & 79,296 & 559 & $\begin{array}{l}0.801 \\
(0.723- \\
0.888)\end{array}$ & 0.001 & $\begin{array}{l}0.854 \\
(0.762- \\
0.958)\end{array}$ & $\begin{array}{l}<.001 \\
0.001\end{array}$ \\
\hline Age group & & & & $\begin{array}{l}< \\
0.001\end{array}$ & & 0.002 \\
\hline$<40$ & 4,565 & 60 & Reference & & Reference & \\
\hline $40-49$ & 13,330 & 155 & $\begin{array}{l}0.922 \\
(0.681- \\
1.247)\end{array}$ & 0.596 & $\begin{array}{l}0.995 \\
(0.701- \\
1.412)\end{array}$ & 0.978 \\
\hline $50-59$ & 33,968 & 362 & $\begin{array}{l}0.953 \\
(0.722- \\
1.256)\end{array}$ & 0.731 & $\begin{array}{l}1.126 \\
(0.817- \\
1.553)\end{array}$ & 0.469 \\
\hline $60-69$ & 41,326 & 385 & $\begin{array}{l}0.913 \\
(0.694- \\
1.201)\end{array}$ & 0.516 & $\begin{array}{l}1.039 \\
(0.755- \\
1.430)\end{array}$ & 0.814 \\
\hline$>69$ & 73,199 & 427 & $\begin{array}{l}0.720 \\
(0.549- \\
0.945)\end{array}$ & 0.018 & $\begin{array}{l}0.839 \\
(0.611- \\
1.152)\end{array}$ & 0.278 \\
\hline Surgery & & & & $\begin{array}{l}<.001 \\
\end{array}$ & & $\begin{array}{l}< \\
0.001\end{array}$ \\
\hline No & 25,682 & 988 & Reference & & Reference & \\
\hline Yes & 140,425 & 398 & $\begin{array}{l}0.378 \\
(0.294- \\
0.486)\end{array}$ & $\begin{array}{l}<.001 \\
0.00\end{array}$ & $\begin{array}{l}0.363 \\
(0.309- \\
0.426)\end{array}$ & $\begin{array}{l}P< \\
0.001\end{array}$ \\
\hline Unknown & 281 & 3 & $\begin{array}{l}0.580 \\
(0.210- \\
1.601)\end{array}$ & 0.293 & $\begin{array}{l}0.618 \\
(0.190- \\
2.004)\end{array}$ & 0.422 \\
\hline T stage & & & & $\begin{array}{l}< \\
0.001\end{array}$ & & \\
\hline Tis, T0,T1,T2 $(0,1,2 \bigotimes 3)$ & 54,934 & 226 & Reference & & & \\
\hline
\end{tabular}

Abbreviations: CRC, colorectal cancer; RCC, right-sided colon cancer; LCC, left-sided colon cancer; RSC, Rectosigmoid cancer; ORs: odds ratios; Cl: confidence interval. 


\begin{tabular}{|c|c|c|c|c|c|c|}
\hline \multirow[t]{2}{*}{ Patient characteristic } & \multicolumn{2}{|l|}{ Patients } & \multicolumn{2}{|c|}{ Among Entire cohort } & \multicolumn{2}{|c|}{$\begin{array}{l}\text { Among cohort with } \\
\text { metastasis }\end{array}$} \\
\hline & Patients & $\begin{array}{l}\text { Bone } \\
\text { metastasis }\end{array}$ & OR (95\%Cl) & $\begin{array}{l}P \\
\text { value }\end{array}$ & $\begin{array}{l}\text { OR } \\
(95 \% \mathrm{Cl})\end{array}$ & $\begin{array}{l}P \\
\text { value }\end{array}$ \\
\hline T3-T4 $(4,5)$ & 94,713 & 627 & $\begin{array}{l}1.230 \\
(1.050- \\
1.442)\end{array}$ & 0.001 & & \\
\hline Unknown & 16,741 & 536 & $\begin{array}{l}1.337 \\
(1.145- \\
1.562)\end{array}$ & 0.01 & & \\
\hline $\mathrm{N}$ stage & & & & $<_{0.001}$ & & $\stackrel{<}{0.001}$ \\
\hline NO & 96,044 & 464 & Reference & & Reference & \\
\hline $\mathrm{N} 1$ & 38,670 & 420 & $\begin{array}{l}1.305 \\
(1.135- \\
1.500)\end{array}$ & $<_{0.001}$ & $\begin{array}{l}1.278 \\
(1.102- \\
1.483)\end{array}$ & 0.001 \\
\hline N2 & 21,119 & 250 & $\begin{array}{l}1.756 \\
(1.421- \\
2.169)\end{array}$ & $<.001$ & $\begin{array}{l}1.548 \\
(1.229- \\
1.949)\end{array}$ & $<.001$ \\
\hline Unknown & 10.555 & 255 & $\begin{array}{l}1.202 \\
(1.031- \\
1.401)\end{array}$ & $<.001$ & $\begin{array}{l}1.289 \\
(1.095- \\
1.517)\end{array}$ & 0.002 \\
\hline Pathology type & & & & $\begin{array}{l}< \\
0.001\end{array}$ & & 0.004 \\
\hline Adenomas & 148,848 & 1,146 & Reference & & Reference & \\
\hline Mucinous & 12,430 & 110 & $\begin{array}{l}1.602 \\
(1.322- \\
1.942)\end{array}$ & $<.001$ & $\begin{array}{l}1.447 \\
(1.142- \\
1.832)\end{array}$ & 0.002 \\
\hline Other type & 3,897 & 93 & $\begin{array}{l}1.322 \\
(1.083- \\
1.615)\end{array}$ & 0.006 & $\begin{array}{l}1.260 \\
(1.003- \\
1.582)\end{array}$ & 0.047 \\
\hline Unspecified & 1,213 & 40 & $\begin{array}{l}1.124 \\
(0.833- \\
1.518)\end{array}$ & 0.445 & $\begin{array}{l}0.907 \\
(0.643- \\
1.280)\end{array}$ & 0.580 \\
\hline Pathology grade & & & & $\begin{array}{l}< \\
0.001\end{array}$ & & $\stackrel{<}{0.001}$ \\
\hline Well differentiated & 15,215 & 48 & Reference & & Reference & \\
\hline
\end{tabular}

Abbreviations: CRC, colorectal cancer; RCC, right-sided colon cancer; LCC, left-sided colon cancer; RSC, Rectosigmoid cancer; ORs: odds ratios; Cl: confidence interval. 


\begin{tabular}{|c|c|c|c|c|c|c|}
\hline \multirow[t]{2}{*}{ Patient characteristic } & \multicolumn{2}{|l|}{ Patients } & \multicolumn{2}{|c|}{ Among Entire cohort } & \multicolumn{2}{|c|}{$\begin{array}{l}\text { Among cohort with } \\
\text { metastasis }\end{array}$} \\
\hline & Patients & $\begin{array}{l}\text { Bone } \\
\text { metastasis }\end{array}$ & OR (95\%Cl) & $\begin{array}{l}P \\
\text { value }\end{array}$ & $\begin{array}{l}\text { OR } \\
(95 \% \mathrm{Cl})\end{array}$ & $\begin{array}{l}\mathrm{P} \\
\text { value }\end{array}$ \\
\hline Moderately differentiated & 101,357 & 526 & $\begin{array}{l}1.196 \\
(0.907- \\
1.575)\end{array}$ & 0.204 & $\begin{array}{l}1.024 \\
(0.744- \\
1.410)\end{array}$ & 0.884 \\
\hline Poorly differentiated & 23.044 & 310 & $\begin{array}{l}2.216 \\
(1.663- \\
2.953)\end{array}$ & $<001$ & $\begin{array}{l}1.655 \\
(1.186- \\
2.309)\end{array}$ & 0.003 \\
\hline Undifferentiated & 4,488 & 48 & $\begin{array}{l}2.305 \\
(1.580- \\
3.362)\end{array}$ & $<0.001$ & $\begin{array}{l}1.913 \\
(1.244- \\
2.941)\end{array}$ & 0.003 \\
\hline Unknown & 22,284 & 457 & $\begin{array}{l}1.483 \\
(1.119- \\
1.964)\end{array}$ & $<.001$ & $\begin{array}{l}1.326 \\
(0.958- \\
1.835)\end{array}$ & 0.089 \\
\hline Number of Lymph node & & & & $<.001$ & & 0.012 \\
\hline 0 & 74,454 & 74 & Reference & & Reference & \\
\hline$<12$ & 44,662 & 202 & $\begin{array}{l}1.559 \\
(1.172- \\
2.072)\end{array}$ & 0.002 & $\begin{array}{l}0.899 \\
(0.638- \\
1.267)\end{array}$ & 0.543 \\
\hline$>=12$ & 3,621 & 76 & $\begin{array}{l}3.129 \\
(2.163- \\
4.525)\end{array}$ & $<0.001$ & $\begin{array}{l}1.391 \\
(0.897- \\
2.158)\end{array}$ & 0.140 \\
\hline Unknown & 43,651 & 1,037 & $\begin{array}{l}1.786 \\
(1.173- \\
2.721)\end{array}$ & 0.007 & $\begin{array}{l}1.459 \\
(0.922- \\
2.309)\end{array}$ & 0.107 \\
\hline Tumor site & & & & 0.001 & & $\begin{array}{l}< \\
0.001\end{array}$ \\
\hline $\mathrm{RCC}$ & & & Reference & & Reference & \\
\hline LCC & & & $\begin{array}{l}1.126 \\
(0.991- \\
1.279)\end{array}$ & 0.068 & $\begin{array}{l}0.979 \\
(0.859- \\
1.116)\end{array}$ & 0.753 \\
\hline RSC & & & $\begin{array}{l}1.633 \\
(1.461- \\
1.825\end{array}$ & $<001$ & $\begin{array}{l}1.600 \\
(1.427- \\
1.795)\end{array}$ & $\begin{array}{l}<.001 \\
0 .\end{array}$ \\
\hline \multicolumn{7}{|l|}{ Brain metastasis } \\
\hline No & 166,004 & 1,322 & & & & \\
\hline
\end{tabular}

Abbreviations: CRC, colorectal cancer; RCC, right-sided colon cancer; LCC, left-sided colon cancer; RSC, Rectosigmoid cancer; ORs: odds ratios; Cl: confidence interval. 


\begin{tabular}{|c|c|c|c|c|c|c|}
\hline \multirow[t]{2}{*}{ Patient characteristic } & \multicolumn{2}{|l|}{ Patients } & \multicolumn{2}{|c|}{ Among Entire cohort } & \multicolumn{2}{|c|}{$\begin{array}{l}\text { Among cohort with } \\
\text { metastasis }\end{array}$} \\
\hline & Patients & $\begin{array}{l}\text { Bone } \\
\text { metastasis }\end{array}$ & OR $(95 \% \mathrm{Cl})$ & $\begin{array}{l}P \\
\text { value }\end{array}$ & $\begin{array}{l}\text { OR } \\
(95 \% \mathrm{Cl})\end{array}$ & $\begin{array}{l}P \\
\text { value }\end{array}$ \\
\hline Yes & 384 & 67 & $\begin{array}{l}2.860 \\
(2.012- \\
4.067)\end{array}$ & $\begin{array}{l}<.001 \\
0.00\end{array}$ & $\begin{array}{l}6.035 \\
(2.848- \\
12.787)\end{array}$ & $<.001$ \\
\hline Lung metastasis & & & & $<.001$ & & 0.001 \\
\hline No & 158,660 & 782 & Reference & & Reference & \\
\hline Yes & 7,206 & 580 & $\begin{array}{l}1.838 \\
(1.519- \\
2.225)\end{array}$ & $<.001$ & $\begin{array}{l}3.881 \\
(1.873- \\
8.040)\end{array}$ & <. \\
\hline Unknown & 522 & 27 & $\begin{array}{l}2.343 \\
(0.952- \\
5.764)\end{array}$ & 0.064 & $\begin{array}{l}3.734 \\
(0.941- \\
14.821)\end{array}$ & 0.061 \\
\hline Liver metastasis & & & & & & 0.107 \\
\hline No & 143,768 & 378 & & & Reference & \\
\hline Yes & 22,336 & 1006 & & & $\begin{array}{l}2.168 \\
(1.055- \\
4.452)\end{array}$ & 0.035 \\
\hline Unknown & 284 & 5 & & & $\begin{array}{l}2.032 \\
(0.585- \\
7.058)\end{array}$ & 0.264 \\
\hline $\begin{array}{l}\text { Extrabone metastasis sites to } \\
\text { brain, lung and liver }\end{array}$ & & & & $<.001$ & & $\begin{array}{l}< \\
0.001\end{array}$ \\
\hline 0 & 141,400 & 243 & Reference & & Reference & \\
\hline 1 & 19,016 & 631 & $\begin{array}{l}3.811 \\
(1.655- \\
8.773)\end{array}$ & 0.002 & $\begin{array}{l}3.826 \\
(1.662- \\
8.808)\end{array}$ & 0.002 \\
\hline 2 & 5,116 & 458 & $\begin{array}{l}6.600 \\
(5.676- \\
7.674)\end{array}$ & $\begin{array}{l}<.001 \\
0.00\end{array}$ & $\begin{array}{l}6.557 \\
(5.641- \\
7.623)\end{array}$ & $<.001$ \\
\hline 3 & 112 & 28 & $\begin{array}{l}8.278 \\
(6.448- \\
10.628)\end{array}$ & $\dot{0} 001$ & $\begin{array}{l}8.191 \\
(6.381- \\
10.514)\end{array}$ & $\begin{array}{l}< \\
0.001\end{array}$ \\
\hline Other organ metastasis & 744 & 29 & $\begin{array}{l}9.061 \\
(4.860- \\
16.895)\end{array}$ & $<.001$ & $\begin{array}{l}8.959 \\
(4.805- \\
16.703)\end{array}$ & <. \\
\hline
\end{tabular}




\section{Survival}

The median survival time (MST) for patients with bone metastasis was 11.3 months (Fig. 1A). For RCC, LCC, and RSC, the MST was 19.9 months, 22.3 months and 22.2 months when bone metastasis was absent, respectively. However, when bone metastasis was present, the MST decreased significantly and was 11.4 months, 12.4 months and 13.0 months for RCC, LCC, and RSC, respectively (Fig. 1B and Table 1). Additionally, patients with bone metastasis were able to benefit from surgery as shown in Fig. 1C.

Multivariable Cox regression (Table 3 ) for OS among patients with bone metastases at diagnosis revealed that older age $(60-69$ vs. $<40, \mathrm{HR}=1.739,95 \% \mathrm{Cl}, 1.226-2.468, \mathrm{P}=0.002 ;>69$ vs. $<40, \mathrm{HR}=2.171,95 \% \mathrm{Cl}=$ $1.53-3.072, \mathrm{P}<0.001$ ), poorly and undifferentiated (poorly vs. well, $\mathrm{HR}=1.451,95 \% \mathrm{Cl}, 1.058-1.990, \mathrm{P}=$ 0.021 ; undifferentiated vs. well, $\mathrm{HR}=1.845,95 \% \mathrm{Cl}, 1.231-2.766, \mathrm{P}=0.003), \mathrm{RCC}$ ( $\mathrm{LCC}$ vs. RCC, HR=0.844, $95 \% \mathrm{Cl}, 0.728-0.978, \mathrm{P}=0.024$; RSC vs. $\mathrm{RCC}, \mathrm{HR}=0.709,95 \% \mathrm{Cl}, 0.622-0.809, \mathrm{P}<0.001$ ), and more extrabone metastasis lesions ( 2 vs. $0, \mathrm{HR}=1.236,95 \% \mathrm{Cl}, 1.050-2.429, \mathrm{P}=0.011 ; 3$ vs. $0, \mathrm{HR}=1.612,95 \% \mathrm{Cl}$, $1.070-2.429, P=0.023$ ) were all significantly associated with poorer OS. CRC-cause mortality among patients with bone metastases at diagnosis is also presented in Table 3 . 
Table 3

Multivariable Cox Regression for All-Cause Mortality and CRC Cancer-Specific Mortality

\begin{tabular}{|c|c|c|c|c|}
\hline \multirow[t]{2}{*}{ Patient characteristic } & \multicolumn{2}{|c|}{ All-cause Mortality } & \multicolumn{2}{|c|}{ CRC-Specific Mortality } \\
\hline & $\mathrm{HR}(95 \% \mathrm{Cl})$ & $\begin{array}{l}\mathrm{P} \\
\text { value }\end{array}$ & $\mathrm{HR}(95 \% \mathrm{Cl})$ & $\begin{array}{l}P \\
\text { value }\end{array}$ \\
\hline Age group & & $<.001$ & & $\begin{array}{l}< \\
0.001\end{array}$ \\
\hline$<40$ & Reference & & Reference & \\
\hline $40-49$ & $\begin{array}{l}1.157(0.791- \\
1.695)\end{array}$ & 0.452 & $\begin{array}{l}1.211(0.805- \\
1.823)\end{array}$ & 0.358 \\
\hline $50-59$ & $\begin{array}{l}1.354(0.951- \\
1.927)\end{array}$ & 0.093 & $\begin{array}{l}1.340(0.913- \\
1.966)\end{array}$ & 0.135 \\
\hline $60-69$ & $\begin{array}{l}1.739(1.226- \\
2.468)\end{array}$ & 0.002 & $\begin{array}{l}1.799(1.229- \\
2.634)\end{array}$ & 0.003 \\
\hline$>69$ & $\begin{array}{l}2.171(1.534- \\
3.072)\end{array}$ & $<.001$ & $\begin{array}{l}2.183(1.493- \\
3.194)\end{array}$ & $\begin{array}{l}< \\
0.001\end{array}$ \\
\hline Surgery & & $<.001$ & & $\begin{array}{l}< \\
0.001\end{array}$ \\
\hline No & Reference & & Reference & \\
\hline Yes & $\begin{array}{l}0.598(0.512- \\
0.698)\end{array}$ & $<.001$ & $\begin{array}{l}0.551(0.461- \\
0.660)\end{array}$ & $\begin{array}{l}< \\
0.001\end{array}$ \\
\hline Unknown & $\begin{array}{l}1.393(0.517- \\
3.757)\end{array}$ & 0.214 & $\begin{array}{l}1.712(0.541- \\
5.417)\end{array}$ & 0.360 \\
\hline $\mathrm{N}$ stage & & $\hat{0}_{0.001}$ & & $\stackrel{<}{0.001}$ \\
\hline NO & Reference & & Reference & \\
\hline N1 & $\begin{array}{l}0.923(0.802- \\
1.063)\end{array}$ & 0.267 & $\begin{array}{l}0.856(0.728- \\
1.008)\end{array}$ & 0.062 \\
\hline N2 & $\begin{array}{l}1.180(0.984- \\
1.414)\end{array}$ & 0.074 & $\begin{array}{l}1.255(1.023- \\
1.539)\end{array}$ & 0.030 \\
\hline Unknown & $\begin{array}{l}1.076(0.920- \\
1.259)\end{array}$ & 0.362 & $\begin{array}{l}1.075(0.897- \\
1.288)\end{array}$ & 0.431 \\
\hline \multicolumn{5}{|l|}{ Diagnosed methods } \\
\hline Other method vs. Biopsy & & $<.001$ & & $\begin{array}{l}<.001 \\
0.00\end{array}$ \\
\hline
\end{tabular}

Abbreviations: CRC, colorectal cancer; RCC, right-sided colon cancer; LCC, left-sided colon cancer; RSC, Rectosigmoid cancer; HRs: hazard ratios; Cl: confidence interval. 


\begin{tabular}{|c|c|c|c|c|}
\hline \multirow[t]{2}{*}{ Patient characteristic } & \multicolumn{2}{|c|}{ All-cause Mortality } & \multicolumn{2}{|c|}{ CRC-Specific Mortality } \\
\hline & $\mathrm{HR}(95 \% \mathrm{Cl})$ & $\begin{array}{l}P \\
\text { value }\end{array}$ & $\mathrm{HR}(95 \% \mathrm{Cl})$ & $\begin{array}{l}P \\
\text { value }\end{array}$ \\
\hline Pathology type & & $\stackrel{<}{<.001}$ & & $\begin{array}{l}< \\
0.001\end{array}$ \\
\hline Adenomas & Reference & & Reference & \\
\hline Mucinous & $\begin{array}{l}1.165(0.954- \\
1.423)\end{array}$ & 0.135 & $\begin{array}{l}1.301(1.039- \\
1.630)\end{array}$ & 0.022 \\
\hline Other type & $\begin{array}{l}1.435(1.168- \\
1.763)\end{array}$ & 0.001 & $\begin{array}{l}1.536(1.204- \\
1.958)\end{array}$ & 0.001 \\
\hline Unspecified & $\begin{array}{l}2.465(1.822- \\
3.334)\end{array}$ & $<.001$ & $\begin{array}{l}2.033(1.399- \\
2.955)\end{array}$ & $\begin{array}{l}<.001 \\
0.001\end{array}$ \\
\hline Pathology grade & & $\overleftarrow{0} .001$ & & $\begin{array}{l}<.001 \\
0.00\end{array}$ \\
\hline Well differentiated & Reference & & Reference & \\
\hline Moderately differentiated & $\begin{array}{l}0.960(0.705- \\
1.308)\end{array}$ & 0.797 & $\begin{array}{l}0.951(0.672- \\
1.345)\end{array}$ & 0.777 \\
\hline Poorly differentiated & $\begin{array}{l}1.451(1.058- \\
1.990)\end{array}$ & 0.021 & $\begin{array}{l}1.477(1.036- \\
2.106)\end{array}$ & 0.031 \\
\hline Undifferentiated & $\begin{array}{l}1.845(1.231- \\
2.766)\end{array}$ & 0.003 & $\begin{array}{l}2.199(1.385- \\
3.493)\end{array}$ & 0.001 \\
\hline Unknown & $\begin{array}{l}1.107(0.809- \\
1.515)\end{array}$ & 0.524 & $\begin{array}{l}1.136(0.800- \\
1.614)\end{array}$ & 0.475 \\
\hline Tumor site & & $<001$ & & $\stackrel{<}{0.001}$ \\
\hline $\mathrm{RCC}$ & Reference & & Reference & \\
\hline LCC & $\begin{array}{l}0.844(0.728- \\
0.978)\end{array}$ & 0.024 & $\begin{array}{l}0.857(0.724- \\
1.016)\end{array}$ & 0.075 \\
\hline RSC & $\begin{array}{l}0.709(0.622- \\
0.809)\end{array}$ & ${ }_{0.001}^{<}$ & $\begin{array}{l}0.733(0.631- \\
0.850)\end{array}$ & $\stackrel{<}{0.001}^{-0}$ \\
\hline Liver metastasis & & & & 0.001 \\
\hline Yes & & & Reference & \\
\hline No & & & $\begin{array}{l}1.227(1.060- \\
1.421)\end{array}$ & 0.006 \\
\hline Unknown & & & $\begin{array}{l}4.792(1.748- \\
13.134)\end{array}$ & 0.002 \\
\hline
\end{tabular}




\begin{tabular}{|c|c|c|c|c|}
\hline \multirow[t]{2}{*}{ Patient characteristic } & \multicolumn{2}{|c|}{ All-cause Mortality } & \multicolumn{2}{|c|}{ CRC-Specific Mortality } \\
\hline & $\mathrm{HR}(95 \% \mathrm{Cl})$ & $\begin{array}{l}P \\
\text { value }\end{array}$ & $\mathrm{HR}(95 \% \mathrm{Cl})$ & $\begin{array}{l}P \\
\text { value }\end{array}$ \\
\hline $\begin{array}{l}\text { Extrabone metastasis sites to brain, lung } \\
\text { and liver }\end{array}$ & & 0.008 & & 0.012 \\
\hline 0 & Reference & & Reference & \\
\hline 1 & $\begin{array}{l}1.029(0.882- \\
1.201)\end{array}$ & 0.713 & $\begin{array}{l}1.167(0.887- \\
1.535)\end{array}$ & 0.271 \\
\hline 2 & $\begin{array}{l}1.236(1.050- \\
1.457)\end{array}$ & 0.011 & $\begin{array}{l}1.309(1.129- \\
1.517)\end{array}$ & $<_{0.001}^{<}$ \\
\hline 3 & $\begin{array}{l}1.612(1.070- \\
2.429)\end{array}$ & 0.023 & $\begin{array}{l}1.855(1.509- \\
2.281)\end{array}$ & $\dot{<} 001$ \\
\hline Other organ metastasis 4 & $\begin{array}{l}1.141(0.780- \\
1.670)\end{array}$ & 0.495 & $\begin{array}{l}1.044(0.776- \\
1.405)\end{array}$ & 0.775 \\
\hline
\end{tabular}

We then compared the MST by extent of metastasis sites (Fig. 1D). In general, patients with more metastasis sites had poorer survival. However, there was no survival difference for those with two or three extra-bone metastasis sites $(P=0.336)$. Then, we further compared the MST by subtype as stratified by tumor location and ascertained the extent of systemic disease (Table 4). We also found that the presence of bone metastases at initial diagnosis was associated with shorter survival time compared with patients presenting with 1 metastatic site without baseline bone involvement, except in RCC with brain metastasis (Table 4). There was no survival difference for single brain metastasis (MST $=8.9$ ) and both brain and bone metastasis (MST = 9.0). Notably, for RSC with single brain metastasis, the MST was 14.0 months and then suddenly decreased to 1 month. 
Table 4

The median survival of metastasis CRC with single metastatic site and multiple-sites

\begin{tabular}{lll} 
Subtype & Survival, Median (IOQ), month & \\
\cline { 2 - 2 } & Extra bone metastasis Disease & Extra bone metastasis and Bone \\
Only & Metastases
\end{tabular}

All cohort

Subsite of

metastasis

\begin{tabular}{lll}
0 & $45.8(45.67-45.93)$ & $13.98(11.74-16.21)$ \\
\hline Brain & $12.62(9.25-16)$ & $6.5(1.19-11.81)$ \\
\hline Lung & $24.04(22.92-25.15)$ & $14.31(11.26-17.35)$ \\
\hline Liver & $21.30(20.94-21.66)$ & $11.28(9.97-12.58)$
\end{tabular}

Two sites

\begin{tabular}{lll} 
Lung and liver & $14.78(14.22-15.31)$ & $9.53(8.43-10.64)$ \\
\hline Lung and brain & $7.75(5.25-10.25)$ & $5.82(1.96-9.66)$ \\
\hline Liver and brain & $7.79(4.98-10.60)$ & $8.02(2.75-13.29)$
\end{tabular}

Three sites

$\begin{array}{lll}\text { Lung, liver, and brain } & 6.10(3.74-8.26) & 6.98(4.27-9.69) \\ \text { Other sites } & 16.63(18.21-18.21) & 9.54(4.75-14.32)\end{array}$

RCC

Subsite of

metastasis

\begin{tabular}{lll}
0 & $43.96(43.76-44.16)$ & $10.37(7.33-13.41)$ \\
Brain & $8.94(4.43-13.44)$ & $9(0-18.02)$ \\
\hline Lung & $19.81(17.86-21.78)$ & $12.13(8.27-15.99)$ \\
Liver & $17.06(16.55-17.57)$ & $7.85(6.54-9.15)$
\end{tabular}

Two sites
Lung and liver
$11.41(10.65-12.17)$
$6.79(5.50-8.09)$
Lung and brain
$9.15(2.48-15.83)$
$6.43(1.34-11.50)$
Liver and brain
$6.65(3.52-9.78)$
$7.14(0.62-13.66)$

Abbreviations: CRC, colorectal cancer; RCC, right-sided colon cancer; LCC, left-sided colon cancer; RSC, Rectosigmoid cancer. 


\begin{tabular}{lll} 
Subtype & \multicolumn{2}{l}{ Survival, Median (IOQ), month } \\
\cline { 2 - 2 } & $\begin{array}{ll}\text { Extra bone metastasis Disease } \\
\text { Only }\end{array}$ & $\begin{array}{l}\text { Extra bone metastasis and Bone } \\
\text { Metastases }\end{array}$
\end{tabular}

Three sites

\begin{tabular}{lll} 
Lung, liver, and brain & $6.93(4.93-8.83)$ & $6.5(2.72-10.28)$ \\
\hline Other sites & $14.04(11.84-16.25)$ & $7.46(2.59-12.33)$ \\
\hline LCC & &
\end{tabular}

Subsite of

metastasis

\begin{tabular}{lll}
\hline & $47.00(46.77-47.24)$ & $12.78(8.69-16.87)$ \\
\hline Brain & $13.99(8.11-19.88)$ & $1(0-1)$ \\
\hline Lung & $25.01(22.71-27.31)$ & $14.11(7.14-21.08)$ \\
\hline Liver & $24.29(23.61-24.96)$ & $13.02(10.10-15.93)$ \\
\hline Two sites & & $11.33(8.78-13.87)$ \\
Lung and liver & $15.47(14.44-16.49)$ & $9(0-20.76)$ \\
\hline Lung and brain & $8.04(3.83-12.25)$ & $2.5(0.39-4.61)$
\end{tabular}

Three sites

$\begin{array}{lll}\text { Lung, liver, and brain } & 2.56(1.67-3.45) & 1.67(1.01-2.33) \\ \text { Other sites } & 18.76(15.66-21.87) & 9.88(0-22.41)\end{array}$

RSC

Subsite of metastasis

\begin{tabular}{|lll|}
\hline 0 & $47.24(47.03-47.46)$ & $15.16(12.02-18.30)$ \\
\hline Brain & $15.39(10.16-20.61)$ & $3.8(0-7.89)$ \\
\hline Lung & $26.06(24.44-27.68)$ & $15.56(10.98-20.15)$ \\
\hline Liver & $24.49(23.77-25.21)$ & $13.72(11.21-16.22)$ \\
\hline Two sites & & \\
\hline Lung and liver & $17.31(16.32-18.31)$ & $10.15(8.53-11.78)$ \\
\hline
\end{tabular}

Abbreviations: CRC, colorectal cancer; RCC, right-sided colon cancer; LCC, left-sided colon cancer; RSC, Rectosigmoid cancer. 


\begin{tabular}{|c|c|c|}
\hline \multirow[t]{2}{*}{ Subtype } & \multicolumn{2}{|l|}{ Survival, Median (IOQ), month } \\
\hline & $\begin{array}{l}\text { Extra bone metastasis Disease } \\
\text { Only }\end{array}$ & $\begin{array}{l}\text { Extra bone metastasis and Bone } \\
\text { Metastases }\end{array}$ \\
\hline Lung and brain & $7.39(3.94-10.84)$ & $7.01(5.06-8.94)$ \\
\hline Liver and brain & $15(3.80-26.20)$ & $11.98(4.98-18.99)$ \\
\hline \multicolumn{3}{|l|}{ Three sites } \\
\hline Lung, liver, and brain & $9.77(5.98-13.56)$ & $9.82(5.33-14.29)$ \\
\hline Other sites & $18.61(15.56-21.65)$ & $11.15(4.91-17.39)$ \\
\hline
\end{tabular}

When patients had metastasis at two sites, such as liver and lung metastasis, other bone metastasis was associated with poorer survival except in those with LCC. Patients with liver, brain, and bone metastasis in the LCC, had a MST of 2.5 months but the MST increased to 13.0 months when bone metastasis was not present.

For patients with three organs affected by metastasis, survival difference was independent of bone metastasis.

\section{Discussion}

Similar to previous reports, our study showed that the rate of bone metastasis from CRC was extremely low $[19,20]$. In this study, we summarized the clinicopathological features associated with bone metastasis and prognostic factors for bone metastasis. Especially noteworthy was our finding that RCC is a more significant risk factor for bone metastasis. Bone metastases were also associated with the presence of lung metastases.

Bone is a common site of metastasis from malignant solid tumors such as prostate, breast, kidney, lung, and thyroid cancers[21]. In contrast, metastases to bone in CRC patients account for less than $10 \%[22]$. This suggests that bone metastasis in CRC behaves differently compared with other cancers. Reports have demonstrated that the environment of a specific organ can influence tumor cell adhesion contributing to the efficacy of tumor spread[23, 24]. Liver and lungs are the most frequently affected organs in CRC. As reported in the literature, the first sites of metastases in CRC tend to be the liver or lungs, which both contain dense capillary beds that can trap tumor cells and seed into these organs[24].

Several interesting observations emerge from our analysis of the relationship between lung metastases and RSC with bone metastases. Previous reports have supported a high frequency of lung metastases with brain metastases[25, 26]. However, the relationship has not been as clearly defined with bone metastases even though metachronous pulmonary metastases have been shown be an independent risk factor of bone metastases[27]. These observations may relate to a longer survival associated with lung metastases. Our series confirms previous observations of an increased incidence of bone metastases in patients with RSC[25, 
28]. A previous study reported that a higher incidence of lung metastases was observed in RSC compared with other colon cancers[29]. Thus, we speculated that RSC patients with lung metastases are at particularly high risk of developing bone metastasis.

Our research highlights the differences in OS based on metastatic sites and the number of disease sites at the time of initial diagnosis. We found that the OS of patients with $\mathrm{MCRC}$ varied by location of the metastatic disease and the metastatic number at the time of initial diagnosis. Potential reasons for the survival difference among the various groups may be due to treatment differences associated with various groups and the metastasis effects on critical organ function (e.g., brain metastasis). The median survival for patients with bone metastasis was 11.3 months, which is consistent with Santini et al[30]. These patients showed poorer prognoses than patients with metastasis to other organs. One of the reasons for the very poor prognosis may be that 80 to $90 \%$ of patients with bone metastasis from CRC also have extra-bone metastasis; often in the lung and/or liver[12]. It is thus important to be alert for bone metastasis during surveillance of patients with lung or liver metastases.

Treatment options for bone metastasis are limited; the most used methods include radiotherapy and bisphosphonate, which appear to be only effective in cases diagnosed early. Consequently, early diagnosis is very important in mitigating against high-risk of bone metastasis.

There are several weaknesses in our study. First, the study was retrospective in design and we did not collect detailed information on treatment methods, which could influence prognosis. This may be a confounding factor in our results. Another confounding factor is the bone metastasis number and the location of bone metastasis, which needs further study.

\section{Conclusions}

We reported the incidence of bone metastasis from CRC[19, 20]. Especially noteworthy is our finding that RSC and the presence of more metastasis sites is a significant risk factor for bone metastasis. Unlike other solid tumors, patients with bone metastasis had poorer survival and the MST was significantly shortened for single metastasis disease or when lung and liver metastasis were present.

\section{Abbreviations}

CRC: Colorectal cancer; SREs: Skeletal-related events; mCRC: metastasis colorectal cancer; SEER: Surveillance, Epidemiology, and End Results; RCC: Right-sided location cancer; LCC: Left-sided colon cancer; RSC: rectosigmoid cancer; MC: mucinous adenocarcinoma; AC: adenocarcinoma; OS: Overall Survival; MST: Median Survival Time.

\section{Declarations}

\section{Ethics approval and consent to participate}

Ethical approval was obtained from the institution through the respective institutional review boards, which belong to the Ethics Committee of Sun Yat-sen University Cancer Center. All patients provided written informed 
consent to participate in this study.

\section{Consent for publication}

Not applicable

\section{Availability of data and material}

Raw data was deposited in the Research Data Deposit system (http://www.researchdata.org.cn) of Sun Yatsen University Cancer and can be obtained from the corresponding authors on reasonable request.

\section{Funding}

This work was supported by the National Natural Science Foundation of China (Grants no. 81272641). We would like to thank the native English speaking scientists of Elixigen Company (Huntington Beach, California) for editing our manuscript.

\section{Competing Interests}

The authors have declared that no competing interest exists.

\section{Authors' contributions}

Conceptualization, YL, ZB, and LSS; Methodology, YL, WDH; Software and data curation, YLهHWZ ; Formal Analysis, LSS, JC, XQK; XLP revised it critically for important intellectual content; Writing-Original Draft Preparation, YL; Project Administration and Funding Acquisition, XLP. All authors (YL, LSH, XQK, HWZ, JC, ZB, WDH, XLP) have read and approved the final manuscript.

\section{Acknowledgements}

Not applicable

\section{References}

\section{References:}

1. van der Stok EP, Spaander M, Grunhagen DJ, Verhoef C, Kuipers EJ. Surveillance after curative treatment for colorectal cancer. NAT REV CLIN ONCOL. 2017; 14: 297-315.

2. Jemal A, Ward EM, Johnson CJ, Cronin KA, Ma J, Ryerson B, et al. Annual Report to the Nation on the Status of Cancer, 1975-2014, Featuring Survival. J Natl Cancer Inst. 2017; 109.

3. Ando K, Oki E, Saeki H, Kasagi Y, Tsuda Y, Zaitsu Y, et al. Number of Lymph Node Metastases May Indicate the Regimen for Adjuvant Chemotherapy in Patients with Stage III Colorectal Cancer. ANTICANCER RES. 2015; 35: 6207-6211.

4. Hayat MJ, Howlader N, Reichman ME, Edwards BK. Cancer statistics, trends, and multiple primary cancer analyses from the Surveillance, Epidemiology, and End Results (SEER) Program. ONCOLOGIST. 2007; 12: 20-37. 
5. Galizia G, Lieto E, Orditura M, Castellano P, Imperatore V, Pinto M, et al. First-line chemotherapy vs bowel tumor resection plus chemotherapy for patients with unresectable synchronous colorectal hepatic metastases. Arch Surg. 2008; 143: 352-358, 358.

6. de Mestier L, Manceau G, Neuzillet C, Bachet JB, Spano JP, Kianmanesh R, et al. Primary tumor resection in colorectal cancer with unresectable synchronous metastases: A review. World J Gastrointest Oncol. 2014; 6: 156-169.

7. Rotolo N, De Monte L, Imperatori A, Dominioni L. Pulmonary resections of single metastases from colorectal cancer. SURG ONCOL. 2007; 16 Suppl 1: S141-S144.

8. Edge SB, Sobin LH, Page DL, Gospodarowicz MK, Greene FL, Winchester DP. Re: Colon cancer survival rates with the new American Joint Committee on Cancer sixth edition staging. J Natl Cancer Inst. 2005; 97: 463-464, 464-465.

9. Coleman RE. Skeletal complications of malignancy. CANCER-AM CANCER SOC. 1997; 80: $1588-1594$.

10. Nozue M, Oshiro Y, Kurata M, Seino K, Koike N, Kawamoto T, et al. Treatment and prognosis in colorectal cancer patients with bone metastasis. ONCOL REP. 2002; 9: 109-112.

11. Heras P, Karagiannis S, Kritikos K, Hatzopoulos A, Mitsibounas D. Ibandronate is effective in preventing skeletal events in patients with bone metastases from colorectal cancer. Eur J Cancer Care (Engl). 2007; 16: 539-542.

12. Roth ES, Fetzer DT, Barron BJ, Joseph UA, Gayed IW, Wan DQ. Does colon cancer ever metastasize to bone first? a temporal analysis of colorectal cancer progression. BMC CANCER. 2009; 9: 274.

13. Khattak MA, Martin HL, Beeke C, Price T, Carruthers S, Kim S, et al. Survival differences in patients with metastatic colorectal cancer and with single site metastatic disease at initial presentation: results from South Australian clinical registry for advanced colorectal cancer. Clin Colorectal Cancer. 2012; 11: 247254.

14. Grothey A, Sargent D, Goldberg RM, Schmoll HJ. Survival of patients with advanced colorectal cancer improves with the availability of fluorouracil-leucovorin, irinotecan, and oxaliplatin in the course of treatment. J CLIN ONCOL. 2004; 22: 1209-1214.

15. Tournigand C, Andre T, Achille E, Lledo G, Flesh M, Mery-Mignard D, et al. FOLFIRI followed by FOLFOX6 or the reverse sequence in advanced colorectal cancer: a randomized GERCOR study. J CLIN ONCOL. 2004; 22: 229-237.

16. Rabeneck L, Davila JA, El-Serag HB. Is there a true "shift" to the right colon in the incidence of colorectal cancer? AM J GASTROENTEROL. 2003; 98: 1400-1409.

17. Liang PS, Mayer JD, Wakefield J, Ko CW. Temporal Trends in Geographic and Sociodemographic Disparities in Colorectal Cancer Among Medicare Patients, 1973-2010. J RURAL HEALTH. 2017; 33: 361370.

18. Zhang X, Zhang MJ, Fine J. A proportional hazards regression model for the subdistribution with rightcensored and left-truncated competing risks data. STAT MED. 2011; 30: 1933-1951.

19. O'Connell JB, Maggard MA, Ko CY. Colon cancer survival rates with the new American Joint Committee on Cancer sixth edition staging. J Natl Cancer Inst. 2004; 96: 1420-1425. 
20. Sundermeyer ML, Meropol NJ, Rogatko A, Wang H, Cohen SJ. Changing patterns of bone and brain metastases in patients with colorectal cancer. Clin Colorectal Cancer. 2005; 5: 108-113.

21. Buckwalter JA, Brandser EA. Metastatic disease of the skeleton. AM FAM PHYSICIAN. 1997; 55: 17611768.

22. Katoh M, Unakami M, Hara M, Fukuchi S. Bone metastasis from colorectal cancer in autopsy cases. J GASTROENTEROL. 1995; 30: 615-618.

23. Schluter K, Gassmann P, Enns A, Korb T, Hemping-Bovenkerk A, Holzen J, et al. Organ-specific metastatic tumor cell adhesion and extravasation of colon carcinoma cells with different metastatic potential. AM J PATHOL. 2006; 169: 1064-1073.

24. Chambers AF, Groom AC, MacDonald IC. Dissemination and growth of cancer cells in metastatic sites. NAT REV CANCER. 2002; 2: 563-572.

25. Lee KC. Brain metastases from colorectal carcinoma: the long term survivors. CANCER-AM CANCER SOC. 1997; 79: 1842.

26. Ko FC, Liu JM, Chen WS, Chiang JK, Lin TC, Lin JK. Risk and patterns of brain metastases in colorectal cancer: 27-year experience. DIS COLON RECTUM. 1999; 42: 1467-1471.

27. Li A, Tan Z, Fu C, Wang H, Yuan J. [Analysis of risk factors for bone metastasis after radical resection of colorectal cancer within 5 years]. Zhonghua Wei Chang Wai Ke Za Zhi. 2017; 20: 58-61.

28. Besbeas S, Stearns MJ. Osseous metastases from carcinomas of the colon and rectum. DIS COLON RECTUM. 1978; 21:266-268.

29. Pihl E, Hughes ES, McDermott FT, Johnson WR, Katrivessis H. Lung recurrence after curative surgery for colorectal cancer. DIS COLON RECTUM. 1987; 30: 417-419.

30. Santini D, Tampellini M, Vincenzi B, Ibrahim T, Ortega C, Virzi V, et al. Natural history of bone metastasis in colorectal cancer: final results of a large Italian bone metastases study. ANN ONCOL. 2012; 23: 20722077.

\section{Figures}


A

Patients with bone metastasis

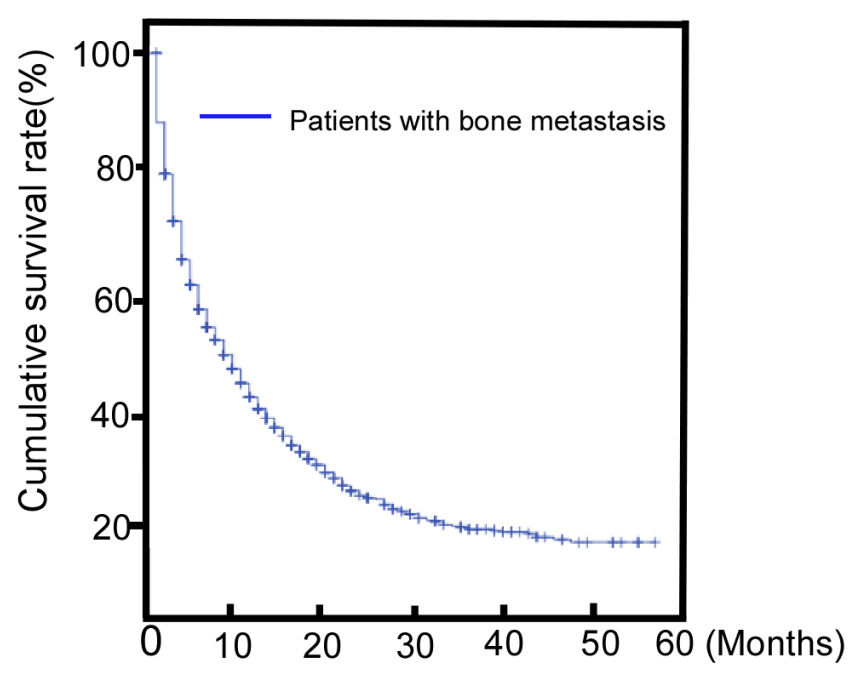

C Patients with bone metastasis

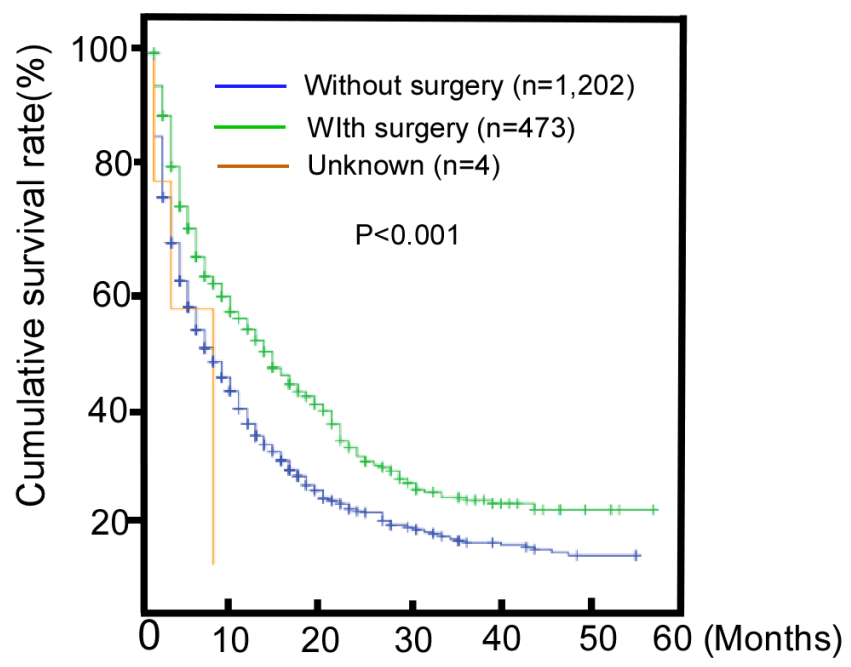

B

Patients with bone metastasis

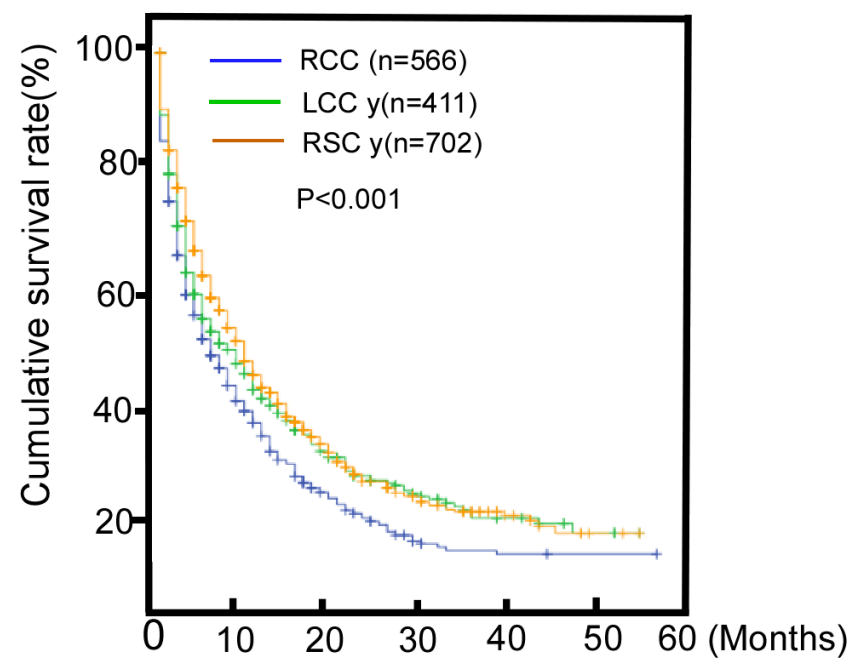

D

\section{Patients with bone metastasis}

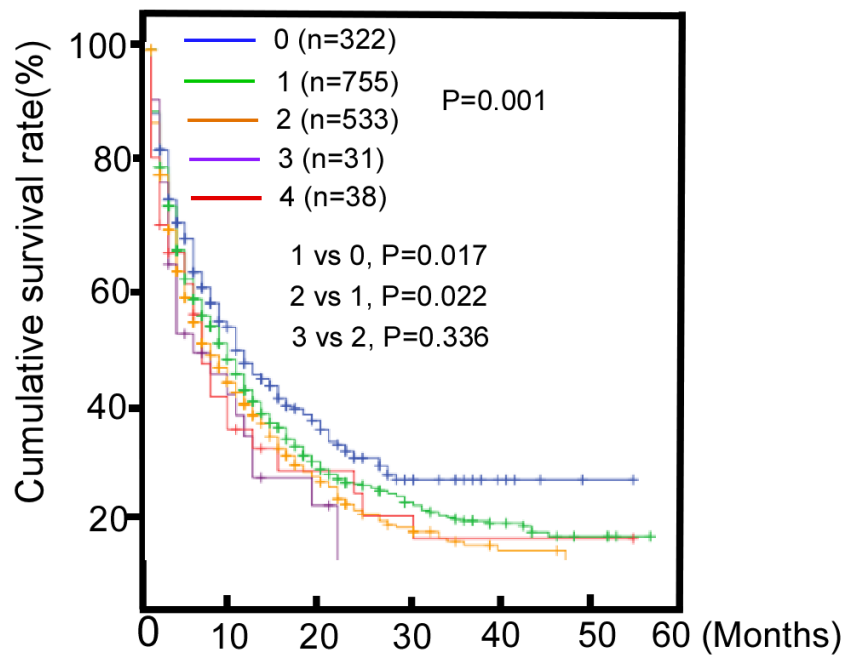

Figure 1

A, Overall survival among patients with bone metastasis; B, Survival difference stratified by subtype in cohort with bone metastasis (RCC, right-sided colon cancer; LCC, Left-sided colon cancer; RSC, Rectosigmoid Cancer); C, The cohort benefit associated with bone metastasis from surgery; D, Survival difference stratified by metastasis number of extra-bone metastases ( 0 , Patients with only BM; 1 , Patients with 1 extra-bone metastasis site; 2 , Patients with 2 extra-bone metastasis sites; 3 , Patients with 3 extra-bone metastasis sites and 4 , Patients with 4 extra-bone metastasis sites). 